\title{
NBSIR 82-2628
}

\section{On the Energy-Release Rate Associated With Diffusional Crack Growth}

U.S. DEPARTMENT OF COMMERCE

National Bureau of Standards

National Measurement Laboratory

Center for Materials Science

Inorganic Materials Division

Washington, DC 20234

December 1982

NBS-DOE Contract No. DE-A105-800R20679

Prepared for

Department of Energy

QC \& \&D Fossil Energy Materials Program

100 ishington, DC 

ON THE ENERGY-RELEASE RATE

ASSOCIATED WITH DIFFUSIONAL CRACK GROWTH

U.S. DEPARTMENT OF COMMERCE

National Bureau of Standards

National Measurement Laboratory

Center for Materials Science

Inorganic Materials Division

Washington, DC 20234

December 1982

NBS-DOE Contract No. DE-A105-800R20679

Prepared for

Department of Energy

AR\&TD Fossil Energy Materials Program

Washington, DC

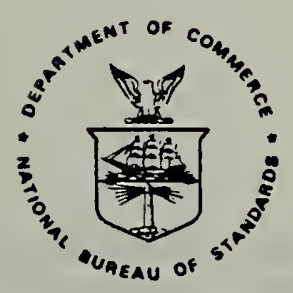

U.S. DEPARTMENT OF COMMERCE, Malcolm Baldrige, Secretary NATIONAL BUREAU OF STANDARDS, Ernest Ambler, Director 

ON THE ENERGY-RELEASE RATE ASSOCIATED WITH DIFFUSIONAL CRACK GROWTH*

\author{
Tze-jer Chuang \\ Center for Materials Science \\ National Bureau of Standards \\ Washington, DC 20234
}

*Work supported by U.S. Department of Energy AR\&TD Fossil Energy Materials

Program under interagency agreement DE-A105-800R20679 with NBS. 



\section{ABSTRACT}

A general expression for the energy release rate ( $G$ ) that arise during steady state crack propagation by diffusion is derived from the standpoint of irreversible thermodynamics. Three contributing components of $\mathrm{G}$ are identified: (i) the Griffith energy $\left(G_{G r}\right)$; (ii) heat generated in the process of surface diffusion; and (iii) grain-boundary diffusion. Further, the total $G$ is shown to be directly related to the well-known J-integral if formulated in the framework of finite deformation elasticity. This expression for $G$ is valid in general even if the response of the material is not linear and the mass transport kinetics does not follow Fick's law. Quantitative evaluations of each component are made for the linear case where field solutions are available. The results show that component (ii) is approximately equal to $G_{G r}$ and is independent of the crack velocity ( $U$ ) whereas component (iii) is a monotonically increasing function with $G$ starting from $0.85 G_{G r}$ when $U$ is at threshold value; and that strain energy contributions can be neglected leading to $G=J=\left(1-v^{2}\right) K^{2} / E$. This means that $G$ is not primarily associated with the release of the strain energy rather, it stems mostly from the negative work done by the normal stresses on the thickening of the grain boundary due to non-uniform deposition of matter along it. 



\section{Introduction}

This paper aims to investigate energy release rates that accompany with diffusive crack growth. Crack-like cavities at grain boundaries are frequently observed in creep ruptured specimens of crystalline solids. Their growth can be attributed to a mechanism involving coupled crack surface and grain-boundary self-diffusion. Chuang et al. ${ }^{1}$ reviewed the subject of diffusive cavitation along grain interfaces and gave the conditions under which the growth of crack-like cavities prevails. In general, creep cavities favor crack-like (slit) shapes when the ratios of applied stress to capillary stress and grain-boundary diffusivity to surface diffusivity are high (say $\gg 1)$ and when the service time approaches the later stages in the growth phase. Under these circumstances, the crack travels in a steady-state fashion at a moderate velocity along the grain boundary. It is then appropriate to treat the crack as semi-infinite, growing at a constant speed in an infinite elastic bicrystal under plane-strain conditions. This case has been considered by Chuang ${ }^{2}$ who solved the coupled problem of diffusion and elastic deformation leading to a specific kinetic law for subcritical crack growth. Although the energy release rate in that case was discussed briefly in Ref. 2 , the present paper attempts to extend the analysis more thoroughly from the standpoint of irreversible thermodynamics. It will be shown that, under steady state, the energy release rates are related to the total entropy production and can be expressed in terms of the well known path-independent J-integral regardless of the form of phenomenological law describing the mass transport processes. For completeness, we also include the strain energy contribution in the formulation. Linearized phenomenological equations of diffusion are then assumed in order to evaluate each individual contribution 
at crack surfaces and grain boundary. It is shown that whereas the exact energy rate quantities are not known, the results presented here always exceed that exact values but no more than $9 \%$.

It seems restrictive to limit the analysis to steady state. However, it is worthwhile to point out that whenever steady state conditions do not hold, no unique energy release rate $e^{3-4}$ can be defined (i.e. it becomes time-dependent). Hence, it is meaningless to discuss the subject of energy release rate without imposition of steady-state conditions.

\section{Thermodynamic Formulation}

The energy release rate is derived in this section from the approach of non-equilibrium thermodynamics. For simplicity, but without loss of generality, consider a single component system in diathermal contact with an environment. Then the entropic fundamental equation has the form $s=s(e, n)$ where $s$, e and $\mathbf{n}$ are respectively the entropy, internal energy and number of atoms per unit volume. Writing $e=e(s, n)$, it can be shown that

$$
\mathrm{ds}=\frac{1}{\mathrm{~T}} \mathrm{de}-\frac{\mu}{\mathrm{T}} \mathrm{dn}
$$

where $T=\left(\frac{\partial e}{\partial s}\right) n, \mu=\left(\frac{\partial e}{\partial n}\right) s$ are the temperature and chemical potential respectively. These intensive parameters are defined strictly under thermodynamic equilibrium conditions. However, in a nonequilibrium system such as a solid undergoing diffusional crack growth, $T$ and $\mu$ are permitted to 
vary continuously throughout the body under the implicit assumption that the functions $T=T(n, s), \mu=\mu(n, s)$ remain the same as they would in local equilibrium. It is then logical to define the entropy flux (and hence the heat flux as well) consistent with Eq. (1) ${ }^{5}$ :

$$
\underline{J}_{Q} \equiv T \underline{J}_{S}=\underline{J}_{e}-\mu \underline{J}
$$

where $\mathrm{Je}$ and $\underline{\mathrm{J}}$ are the energy and matter fluxes respectively.

The conservation law dictates that the internal energy and the mass must be conserved while allowing entropy sources (or sinks) to be present. This means that

$$
\begin{aligned}
& 0=\frac{\partial e}{\partial t}+\underline{\nabla} \cdot \underline{J}_{S} \\
& 0=\frac{\partial n}{\partial t}+\underline{\nabla} \cdot \underline{J}
\end{aligned}
$$

and

$$
\dot{s}=\frac{\partial s}{\partial t}+\underline{\nabla} \cdot \underline{J}_{S}
$$

where $\dot{s}$ is the local entropy production rate. It is possible to express $\dot{s}$ in terms of summation of terms involving individual flux and its corresponding intensive parameter as follows

$$
\begin{aligned}
\dot{s} & =\frac{\partial s}{\partial t}+\underline{\nabla} \cdot \underline{J}_{S} \\
& =\frac{1}{T} \frac{\partial e}{\partial t}-\frac{\mu}{T} \frac{\partial n}{\partial t}+\underline{\nabla} \cdot \frac{1}{T}(\underline{J} e-\mu J) \\
& =\underline{J} e \cdot \nabla \frac{1}{T}-\underline{J} \cdot \nabla \frac{\mu}{T} \\
& =\left(\underline{J}_{Q}+\mu \underline{J}\right) \cdot \underline{\nabla} \frac{1}{T}-\mu \underline{J} \cdot \underline{\nabla} \frac{1}{T}-\frac{1}{T} \underline{J} \cdot \underline{\nabla} \mu \\
& =\underline{J}_{Q} \cdot \underline{\nabla} \frac{1}{T}+\frac{\underline{J}}{T} \cdot(-\underline{\nabla} \mu)
\end{aligned}
$$


Hence in general for nonuniform temperature and chemical potential (and hence stress) fields, the entropy production rate consists of two terms:

first, the scalar product of heat flux and gradient of $\left(\frac{1}{T}\right)$, and second, the scalar product of matter flux and negative gradient of $\mu$ divided by the local temperature. Note that at steady state $\underline{\nabla} \cdot \underline{J}_{e}=\underline{\nabla} \cdot \mathrm{J}=0$ and $\underline{\nabla} \cdot \underline{J}_{Q}=$ - $\underline{J} \cdot \underline{\mu} \mu$ according to Eq. (2).

Hence

$$
\dot{\mathrm{s}}=\underline{\nabla} \cdot \frac{\mathrm{J}_{\mathrm{Q}}}{\mathrm{T}}
$$

and for isothermal processes wherein $T=$ constant, the above two equations reduce to

$$
\dot{s}=\frac{1}{T} \underline{J} \cdot(-\underline{\mu})=\frac{1}{T} \underline{\nabla} \cdot \underline{J}_{Q}
$$

We now define $W_{\text {dis }}$, the dissipative work as

$$
\dot{\mathrm{W}}_{\mathrm{dis}}=\mathrm{T} \dot{\mathrm{S}}
$$

where

$$
\dot{\mathrm{s}}=\int_{V} \dot{\mathrm{s}} \mathrm{dV}=\frac{1}{\mathrm{~T}} \int_{V} \underline{\mathrm{J}} \cdot(-\underline{\nabla} \mu) \mathrm{dV}
$$

is the total entropy production rate over the whole body.

We now turn to the first law of thermodynamics which states that the increase in total internal energy of the body must be equal to the total input of the mechanical work done to the body less the heat loss from the body to its surrounding environment through the body's external "skin". This means that 


$$
\dot{E}=\dot{W}-\int_{A} \underline{J}_{Q} \cdot \underline{\underline{D}} \mathrm{dA}
$$

where $A$ represents the collection of external areas in contact with environment where $J_{Q}$ occurs, and $\underline{n}$ is the unit normal vector of A directed outward from the body.

At constant T, Eq. (2) can be integrated over the whole volume to give the following equation in a global form:

$$
-\int_{\mathrm{A}} \underline{J}_{\mathrm{Q}} \cdot \underline{\mathrm{n}} \mathrm{dA}=\mathrm{T} \frac{\partial \mathrm{S}}{\partial t}-\mathrm{T} \dot{\mathrm{S}}
$$

where Eq. (3c) and the Gauss divergence theorem have been used. This equation assures the conservation of heat inside the body. Elimination of the integral in Eqs. (6) and (7) results in

$$
\dot{\mathrm{F}}=\dot{\mathrm{W}}-\mathrm{T} \dot{\mathrm{S}}
$$

where $F \equiv E-T S$ is the total Helmholtz free energy of the body. Eq. (8) shows that the total external work done contributes partially to the increase in the total free energy with the remaining part becoming the dissipative work $\dot{\mathrm{W}}_{\mathrm{dis}}$ since $\dot{\mathrm{W}}_{\mathrm{dis}}=\mathrm{TS}$. Alternatively, we may define the effective heating rate as $\int_{A} J_{Q} \cdot \underline{n} d A$ and this can be shown to be equal to $(\dot{W}-\dot{E})$ from the first law of thermodynamics, Eq. (6).

It will be seen in Sec. 4 that Eqs. (5) and (8) are important in the formulation of the general expression for the energy release rate in which explicit expressions for the thermodynamic parameters in our special case are required. The governing equations leading to those explicit expressions are discussed first in the following section. 


\section{Governing Equations in Diffusive Crack Growth}

Consider a semi-infinite crack travelling along a grain boundary ( $x$-axis) at a constant velocity, $U$ in an infinite bicrystal subject to a remote loading (see Fig. 1). Under the influence of the applied stress, the crack is supposed to advance by matter fluxes induced by stress-assisted self-diffusion along the crack surfaces and grain-boundary. As a result, the grain-boundary is thickened by an amount $\delta(x)$, defined by the discontinuity of vertical displacements in $y, \delta(x) \equiv[u]^{ \pm}=u^{+}(x)-u^{-}(x)$, at the upper and lower surfaces of the grain boundary, due to grain-boundary diffusion. This $\delta(x)$, in turn, produces a misfit residual stress field which upon adding to the applied stress field, yields to the actual stress distribution $\sigma_{y y}(x)$ along the grain boundary. These two functions $\delta(x)$ and $\sigma_{y y}(x)$, illustrated schematically in Fig. 1, have been shown to play an important role in governing the behavior of creep crack growth. 2

Let $u_{n}$ be the normal recession rate at the crack surface; $\theta(s)$ be the angle of the tangent at any point with the vertical y-axis and $s$ the arc length along the curved crack surface as measured from the crack tip (see Fig. 1). Then steady-state conditions require

$$
u_{n}(s)=u \cos \theta(s)
$$

at any point s on the surface. Strictly speaking, the LHS of Eq. (9) should include a term $\underline{U}_{m}$. $\underline{n}$ where $\underline{U}_{m}$ is the velocity vector of the material point and $\underline{n}$ is the unit normal vector of the surface area directed outward from the material. This term $\left(\underline{U}_{m} \cdot \underline{n}\right)$ is important in materials where time-dependent creep deformation takes place. For elastic materials considered here, this 
term can therefore be omitted. The range of $\theta$ in $\mathrm{Eq}$. (9) for all points on the crack surface is such that

$$
\frac{\pi}{2}>\theta>\theta_{\text {tip }}
$$

and when $\theta=\pi / 2$ (i.e. at the center of the crack), $u_{n}=0$.

In Fig. 1, we set up a two dimensional Cartesian coordinate system $(x, y)$ and locate the origin at the moving crack tip. Thus, under steady-state conditions

$$
\partial \delta / \partial t=-u \partial \delta / \partial x
$$

everywhere along the grain boundary.

The explicit expressions for the chemical potentials at the crack surfaces and grain boundary applicable to the case of diffusive cavity growth have been derived by Rice and Chuang ${ }^{3}$ using Herring's procedures. The effects of surface strains were considered and shown to have no influence on the final expressions for chemical potential. The results are quoted here:

$$
\mu_{b}(x)=\Omega w(x)-\Omega \sigma_{y y}(x)
$$

and

$$
\mu_{s}(s)=\Omega w(s)-\Omega \gamma_{s} K(s)
$$


where $\mu_{b}$ and $\mu_{s}$ are the chemical potentials at the grain boundary and crack surfaces respectively; $\Omega$ the atomic volume in the current deformed state; $\gamma_{s}$, surface free energy per unit area of current configuration; $K$, surface curvature, (positive for concave surfaces) and $w$, the strain energy density.

The law of mass conservation asserts that

$$
u_{n}(s)=\Omega \partial J_{s} / \partial s
$$

at the crack surfaces and

$$
\partial \delta / \partial t=\Omega \partial J_{b} / \partial x
$$

at the grain boundary. Also, at the crack tip where $x=0$, we must have, from Eqs. (11),

$$
\begin{aligned}
& \sigma_{t i p}=\gamma_{s} \kappa_{t i p} \\
& \Omega\left(J_{b}\right)_{t i p}=2 \mathrm{hu}
\end{aligned}
$$

and

$$
\left(\mathrm{J}_{\mathrm{b}}\right)_{\text {tip }}=2\left(\mathrm{~J}_{\mathrm{s}}\right)_{\text {tip }}
$$

where $J_{b}(x)$ and $J_{s}(s)$ are the matter fluxes at the grain boundary and crack surfaces respectively; $2 \mathrm{~h}$ is the constant crack thickness (see Fig. 1). For cases where velocity fields are substantial, Chen and Argon ${ }^{6}$ argued that 
instead of Eq. (14c), $2 J_{s}=J_{b}+\delta J$ where $\delta J$ stands for additional surface flux induced by the velocity field (jacking). However, although the velocity field does contribute to the change in volume of the void, it can not alter the balance of matter flux locally at the crack tip. Hence Eq. (14C) is generaly valid regardless of the field of strain rates.

An additional equation can be derived if we assume no local entropy production takes place at the crack tip. The dihedral angle must take a value such that

$$
\operatorname{Sin} \theta_{t i p}=\gamma_{b} / 2 \gamma_{s}
$$

be satisfied. Here $2 \theta_{\text {tip }}=\pi-\psi$, $\psi$ being the dihedral angle at the crack tip and $\gamma_{b}$ the grain-boundary free energy. It is interesting to note that two totally independent approaches, one based on the energy transfer that occurs when matter is removed from the crack surfaces and deposited along the grain boundary ${ }^{3}$, and another ${ }^{4}$, based on invariant dissipative work, both lead to Eq. (15).

Now we can combine Eqs. (10) and (13) to give

$$
\delta(x)=\frac{\Omega}{v} J_{b}(x)
$$

if we assume both $\delta$ and $J_{b}$ decay asymptotically to zero as $x$ approaches infinity (as expected by physical argument). Eq. (16) asserts that the boundary fluxes are linearly dependent on $\delta(x)$ and that at the crack tip 


$$
\delta_{\text {tip }}=\frac{\Omega}{v}\left(\mathrm{~J}_{\mathrm{b}}\right)_{\text {tip }}=2 \mathrm{~h}
$$

according to Eq. (14b).

Finally, the gradient of surface fluxes can be expressed in terms of $\theta$ upon combination of Eqs. (9) and (12):

$$
\partial J_{s} / \partial s=\left(\frac{U}{\Omega}\right) \cos \theta
$$

The governing equations derived in this section are necessary, as will be seen in the next section, for the investigation of energy release rate in diffusive crack growth.

\section{Energy release rate in diffusive crack growth}

It is well known in fracture mechanics that when a crack is propagating in a solid, there is always an energy-release rate accompanying crack growth. Indeed, this provides the thermodynamic "force" driving the crack growth. The conventional energy-release rate $G$ is defined by

$$
U G \equiv-\frac{d P}{d t}=\dot{W}-\frac{d}{d t} \int_{V} w d V
$$

for a class of elastic-brittle cracks without diffusion. Here $P$ is the total potential energy, $w=w(x, y)$ is the strain energy density and the integration is performed over the whole volume of the body. For a sake of consistency we extend this definition to the case of diffusive crack growth by writing 


$$
U G \equiv \dot{W}-\dot{\mathrm{F}}_{\mathrm{v}}
$$

since the time rate of strain energy is identical to that of the Helmholtz free energy for isothermal processes. Here $F_{v}$ represents the total volumetric free energy; it relates to the total free energy of the body by $F=F_{V}+F_{S}$ where $F_{S}$ is the free energy associated with internal surfaces (cavities and interfaces). Thus, by employing eq. (8) derived in Sec. 2

$$
U G=\dot{\mathrm{F}}_{S}+\dot{\mathrm{W}}_{\mathrm{dis}}=\dot{\mathrm{F}}_{\mathrm{S}}+\mathrm{T} \dot{\mathrm{S}}
$$

wherein according to Eq. (5), $\dot{\mathrm{w}}_{\mathrm{dis}}=\int_{\mathrm{V}} \underline{J} \cdot(-\underline{\nabla} \mu) \mathrm{dV}$. This is justified for the special case of diffusive crack growth since in elastic-brittle materials the motion of dislocations is mild and under creep conditions, the quasi-static processes lead to little phonon emission (i.e. negligible dynamic effects). Accordingly, the total dissipative work is predominantly generated by matter fluxes notably along the high diffusivity paths at internal void surfaces and grain interfaces. Thus the contribution of $\mathrm{W}_{\mathrm{dis}}$ by bulk diffusion could also be neglected unless the temperature approaches the materials melting point. Let $\dot{\mathrm{w}}_{\mathrm{dis}}^{s}$ and $\dot{\mathrm{w}}_{\mathrm{dis}}^{\mathrm{g} \cdot \mathrm{b}}$ be the dissipative work generated per unit time at the crack surfaces and along the grain boundary respectively, we can now write $\dot{\mathrm{w}}_{\mathrm{dis}} \cong \dot{\mathrm{w}}_{\mathrm{dis}}^{\mathrm{s}}+\dot{\mathrm{w}}_{\mathrm{dis}}^{\mathrm{g} \cdot \mathrm{b}}$ and Eq. (20) describing the energy release rate becomes

$$
U G=\dot{\mathrm{F}}_{\mathrm{s}}+\dot{\mathrm{H}}_{\mathrm{dis}}^{\mathrm{s}}+\dot{\mathrm{W}}_{\mathrm{dis}}^{\mathrm{g} \cdot \mathrm{b}}
$$

where

$$
\dot{\mathrm{w}}_{\mathrm{dis}} \mathrm{s}=2 \int_{0}^{\infty} \underline{J}_{s} \cdot\left(-\underline{\nabla} \mu_{s}\right) d s
$$


and

$$
\dot{\hat{w}} \begin{aligned}
& g \cdot b \cdot \\
& \text { dis }
\end{aligned}=\int_{0}^{\infty} \underline{J}_{b} \cdot\left(-\nabla_{\mu_{b}}\right) d x
$$

Notice that a factor of 2 appears in eq. (22), reflecting the fact that there are two identical crack surfaces. The increase in surface free energy $\dot{F}_{s}$ due to steady state crack growth along the grain boundary is simply

$$
\dot{\mathrm{F}}_{\mathrm{s}}=u\left(2 \gamma_{\mathrm{s}}-\gamma_{\mathrm{b}}\right)
$$

since by growing a crack of unit length, two unit areas of free surfaces are created at the expense of one unit area of grain boundary. It should be noted that in the absence of diffusion, $\dot{s}=0$ throughout the whole body, our case then reducing to reversible crack growth of the Griffith model. Then $G_{G r}=\dot{\mathrm{F}}_{s} / U=2 \gamma_{s}-\gamma_{b}$ from Eqs. (20) and (24). This means that $\left(2 \gamma_{s}-\gamma_{b}\right)$ is identical to the Griffith energy.

Eq. (21) serves as the starting point for evaluating $G$. We tackle the problem by solving for $\dot{W}_{\text {dis }}$ (eqs. (22) and (23)) explicitly with the assistance of the equations derived in Sec. 3 .

First, we solve for $\dot{\mathrm{W}}_{\mathrm{dis}}^{\mathrm{s}}$ in $\mathrm{Eq} .,(22)$ in the following way:

$$
\begin{aligned}
& \dot{W}_{\text {dis }}^{s}=2 \int_{0}^{\infty} J_{s}\left(-\partial \mu_{s} / \partial s\right) d s \\
& \text { [by Eq. (22)] } \\
& =-2 \int_{0}^{\infty} \frac{\partial}{\partial s}\left(J_{s} \mu_{s}\right) d s+2 \int_{0}^{\infty} \mu_{s}\left(\partial J_{s} / \partial s\right) d s \quad \begin{array}{c}
\text { [integration by } \\
\text { parts] }
\end{array} \\
& =2 \mathrm{~h} u\left(\gamma_{s} \kappa_{t i p}-w_{t i p}\right)+\frac{2 u}{\Omega} \int_{0}^{\infty} \mu_{s} \cos \theta d s \quad \underset{\text { and }(18)]}{[\text { Eqs. (14) }}
\end{aligned}
$$


The integral in the second term of the RHS is

$$
\begin{aligned}
& \int_{0}^{\infty}\left(w \Omega-\gamma_{s} k \Omega\right) \cos \theta d s \\
& =\Omega\left[\int_{0}^{h} w d y-\gamma_{s}\left(1-\sin \theta_{t i p}\right)\right] \\
& =\Omega\left[\int_{0}^{h} w d y-\left(2 \gamma_{s}-\gamma_{b}\right)\right]
\end{aligned}
$$

[by Eq. (15)]

Thus, the surface dissipative work per unit cracked area results in the following expression:

$$
\frac{\dot{\mathrm{w}}_{d i s}^{s}}{u}=2 \mathrm{~h} \gamma_{s} \kappa_{t i p}-\left(2 \gamma_{s}-\gamma_{b}\right)-2 h w_{t i p}+2 \int_{0}^{h} w d y
$$

This means that, in general, the surface dissipative work is a function of crack geometric parameters (such as $2 \mathrm{~h}$ and $\mathrm{K}_{\mathrm{tip}}^{*}$ ), strain energy distribution along the crack surface and the intrinsic surface and interfacial free energies .

Similarly, the rate of grain-boundary dissipative work can be evaluated in the following way

$$
\begin{aligned}
\dot{\mathrm{w}}_{\mathrm{dis}}^{\mathrm{g} \cdot \mathrm{b}} & =\int_{0}^{\infty} \mathrm{J}_{\mathrm{b}}\left(-\partial \mu_{\mathrm{b}} / \partial \mathrm{x}\right) \mathrm{dx} & \text { [by Eq. (23)] } \\
& =\Omega \int_{0}^{\infty} \mathrm{J}_{\mathrm{b}}\left(\partial \sigma_{\mathrm{yy}} / \partial \mathrm{x}\right) \mathrm{dx}-\Omega \int_{0}^{\infty} \mathrm{J}_{\mathrm{b}}(\partial w / \partial \mathrm{x}) \mathrm{dx} & \text { [by Eq. (11a)] } \\
& =u \int_{0}^{\infty} \delta\left(\partial \sigma_{\mathrm{yy}} / \partial \mathrm{x}\right) \mathrm{dx}-u \int_{0}^{\infty} \delta(\partial w / \partial \mathrm{x}) \mathrm{dx} & \text { [by Eq. (16)] }
\end{aligned}
$$


Thus, after integration by parts and noting that $\delta_{\text {tip }}=2 \mathrm{~h}$ from Eq. (17) and $\sigma_{\text {tip }}=\gamma_{s} k_{\text {tip }}$ from Eq. (14a), the grain-boundary dissipative work per unit cracked area takes the form

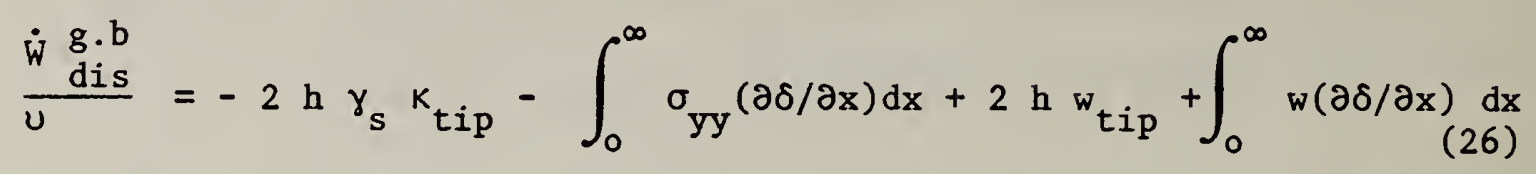

The first term on the RHS of Eq. (26) represents the contribution made by $K^{*}$ tip; the second term is the mechanical work done by $\sigma_{y y}$ on $\delta$, in analogy to the cohesive zone models; the remaining terms are the contributions from the strain energy density.

Substituting Eqs. (24), (25) and (26) into Eq. (21), one obtains the following equation

$$
G=2 \int_{0}^{h} w d y+\int_{0}^{\infty} w(\partial \delta / \partial x) d x-\int_{0}^{\infty} \sigma_{y y}(\partial \delta / \partial x) d x
$$

This is a general expression for Irwin's energy release rate that arises from diffusional crack growth in an (linear or non-linear) elastic solid. We note that in the absence of diffusion, $\delta=0$ and $G=2 \int_{0}^{h} w$ dy becomes the strainenergy release rate appearing in conventional fracture mechanics theory. It can be seen that $G$ is composed of three parts: (1) the loss of strain energy in connection with matter that is removed from the crack surface; (2) the

\footnotetext{
* It might be mentioned here that the curvature cannot be defined exactly at the crack tip mathematically. Hence $k_{t i p}$ is referred to as the curvature at a point immediately adjacent to the crack tip.
} 
gain of strain energy by stressing the deposited matter at the grain boundary to a proper level so as to ensure a coherent fit; and (3) the mechanical work done by normal stresses to allow insertion of the matter which has diffused away from the crack surfaces. We emphasize here that Eq. (27) is derived solely from irreversible thermodynamic principles and therefore is independent of specific constitutive law or phenomenological equations intrinsic to a given material, as long as the behavior of the material remains elastic. Extension of the applicability of Eq. (27) to elastic-plastic materials is possible if one includes also the plastic dissipative work generated by the motion of dislocations in the vicinity of the crack tip as formulated by Thoms on ${ }^{7}$.

It is of special interest to note that an alternative approach from the theory of elasticity can be adopted to derive G. Rice and Drucker ${ }^{8}$, Rice ${ }^{9}$ and Elshelby 10 have shown that the reduction in potential energy for a smooth-surface notch due to removal (or addition) of matter is

$$
-\Delta P=\int_{d V} w d V=\int_{A} w d n d A
$$

where the integral is performed over the volume $\mathrm{dV}$ of the matter being removed (or added). Noting that potential energy is the strain energy minus the mechanical work done (both external and internal), we can rewrite this equation so that it is applicable to the present case as a time rate expression:

$$
\dot{\mathrm{F}}_{\mathrm{v}}-\dot{\mathrm{w}}-\int_{\Gamma_{\mathrm{b}}}\left(-\sigma_{\mathrm{yy}}\right) \dot{\delta} \mathrm{dx}=2 \int_{\Gamma_{s}} w \dot{\delta} \mathrm{ds}+\int_{\Gamma_{\mathrm{b}}} w \dot{\delta} \mathrm{dx}
$$


where $\Gamma_{b}$ and $\Gamma_{s}$ are grain-boundary and crack surface paths respectively; $\dot{\delta}>0$ at $\Gamma_{\mathrm{b}}$ and $\dot{\delta}<0$ at $\Gamma_{\mathrm{s}}$. Notice that this equation neglects the surface tension effects; otherwise an extra term representing the surface work must be added to the LHS $^{3}$.

Now, since along $\Gamma_{s},-\dot{\delta} \mathrm{ds}=u \cos \theta \mathrm{ds}=u$ dy and along $\Gamma_{\mathrm{b}}, \dot{\delta}=-u(\partial \delta / \partial \mathrm{x})$, this equation reduces to

$$
G=\frac{\dot{W}-\dot{F}_{v}}{u}=2 \int_{0}^{h} w d y-\int_{0}^{\infty} \sigma_{y y}\left(\frac{\partial \delta}{\partial x}\right) d x+\int_{0}^{\infty} w\left(\frac{\partial \delta}{\partial x}\right) d x
$$

which is seen to be identical to Eq. (27). Hence, two entirely different derivations, one based on thermodynamic principles and the other on elasticity theory, lead to exactly the same expression for G.

As is well known in linear elastic fracture mechanics theory the path-independent contour integral $\mathrm{J}$ and Irwin's strain energy release rate $\mathrm{G}$ can be shown to be identical, and $G=J=\left(1-v^{2} / E\right) K_{I}{ }^{2}$ where $K_{I}$ is the mode $I$ stress intensity factor, E Youngs modulus and $v$ the Poisson's ratio. Hence it is interesting to connect $G$ and $J$ for diffusive crack growth as considered here.

The definition of $\mathrm{J}$ in the context of finite deformation elasticity is ${ }^{11}$

$$
J \equiv \int_{\Gamma_{0}} w_{0} d Y-\underline{T}_{0} \cdot \partial \underline{u} / \partial X d s_{0}
$$

where $\Gamma_{0}$ is an arbitrary path around the crack tip starting from the lower crack surface and ending at the upper one in a counterclockwise 
direction; $\mathrm{X}$ and $\mathrm{Y}$ are material coordinates in an unstressed state; $\mathrm{dS}_{0}$ is an element of arc length, $W_{0}$ is strain energy per unit volume in undeformed state and $\underline{T}_{0}$ is the nominal stress vector. By taking advantage of the pathindependence property, we can shrink $\Gamma_{0}$ down to the crack lines (i.e. negative X-axis in Fig. 1) and along the flat grain-boundary surfaces (dashed lines in Fig. 1). After a finite deformation into the current deformed state, the portion of $\Gamma_{0}$ along the negative $X$-axis becomes dashed line along the curved "notch surfaces" and the other part of $\Gamma_{0}$ at the grain-boundary surfaces deform into lines following the $\pm \delta / 2$ solutions as shown in Fig. 1 . Then, based on the current configuration, the contribution to $\mathrm{J}$ of the integration along the "notch" surfaces is $\int_{h}^{h} w$ dy since $\underline{T}$ vanishes everywhere there and the contribution made by the terms involving integration along both the upper and lower grain-boundary surfaces is $\int_{0}^{\infty} w(\partial \delta / \partial x) d x-\int_{0}^{\infty} \sigma_{y y}(\partial \delta / \partial x) d x$ since, for integration paths following the $\pm \delta / 2-c u r v e s, d y=-(\partial u / \partial x) d x$ and $\underline{T} \cdot(\partial \underline{u} / \partial s) d s=-\sigma_{y y}(\partial u / \partial x) d x$. Hence

$$
J=\int_{-h}^{h} w d y+\int_{0}^{\infty} w\left(\frac{\partial \delta}{\partial x}\right) d x-\int_{0}^{\infty} \sigma_{y y}\left(\frac{\partial \delta}{\delta x}\right) d x
$$

Upon comparison between this equation and Eq. (27), and noting the symmetry of the upper and lower crack surfaces, we immediately arrive at

$$
G=J
$$

Eq. (28) shows that $G$ in the case of diffusive crack growth is identical to $J$ as in the conventional fracture mechanics expression if one takes finite elastic deformation into account. We emphasize that this expression, Eq. (28) is exact and is applicable to steady-state diffusional crack growth in a 
brittle-elastic material even if the constitutive and phenomenological equations are non-linear. This is because $G$ was derived from energy considerations and while specific forms of stress-strain and diffusion equations can affect field parameters, they do not have any influence on the energy balance.

\section{Discussion}

We have shown from non-equilibrium thermodynamics that the general expression for the energy release rate $G$ that incurs during diffusional crack growth processes is composed of three terms, namely (i) surface energy increase arising from the creation of new crack surfaces as well as dissipative work (heat generated) by diffusional processes along (ii) the crack free surfaces and (iii) the grain boundary. This statement is valid so long as the conditions of steady state and mass and energy conservation are met and no plastic processes take place inside the body. Derivation of $\mathrm{G}$ from the approach of elasticity theory was also given which showed an identical result. In addition, it was shown that $G$ is related directly to the well-known J-integral as given by Eq. (28).

To further investigate each individual component contributing to $G$ the curvature at the crack tip, stress and strain energy fields have to be determined (see Eqs. (25) and (26)). These field parameters must be solved from phenomological equations. Unfortunately, no solutions are available in the general case of a diffusional grain-boundary crack, obeying non-linear diffusion equations and growing in a non-linear elastic material. We therefore limit our discussion to a special linerized case where solutions are 
readily available. The diffusion equations then follow Fick's law and take the form

$$
\underline{J}=\text { (const.) } \underline{\nabla} \mu
$$

where $\underline{J}=\underline{J}_{s}$ and $\mu=\mu_{s}$ at crack surfaces, and $\underline{J}=\underline{J}_{b}$ and $\mu=\mu_{b}$ along the grain boundary. Also the stress-strain relations follow Hooke's law, so the strain energy function takes quadratic form in stress (or strain). Further, the strain energy contribution to $\mu$ (and hence to $G$ ) can be neglected even at the crack tip (e.g. see Eq. (8) of Ref. 3). This is because the radius of curvature developed by surface diffusion at the crack tip is normally large compared to lattice spacing and misfit residual stresses induced by mass transport along the grain boundary create "wedging" effects, thus alleviating the stress concentration near and at the crack tip. Actual stress solutions given by Chuang confirm this assertion. Thus, the version of $\dot{\mathrm{w}}_{\mathrm{dis}}^{\mathrm{s}}$, Eq. (25), $\dot{\mathrm{w}}_{\mathrm{dis}}^{\mathrm{g} \cdot \mathrm{b}}$, Eq. (26) and G Eqs. (27) and (28) in the linerized theory can be rewritten as

$$
\begin{aligned}
& \frac{\dot{\hat{W}}_{d i s}^{s}}{u}=2 h \gamma_{s} k_{t i p}-\left(2 \gamma_{s}-\gamma_{b}\right) \\
& \frac{\dot{\mathrm{w}}_{d i s}^{g \cdot b}}{u}=-2 h \gamma_{s} \kappa_{t i p}-\int_{0}^{\infty} \sigma_{y y}\left(\frac{\partial \delta}{\partial x}\right) d x
\end{aligned}
$$

and

$$
G=J=-\int_{0}^{\infty} \sigma_{y y}\left(\frac{\partial \delta}{\partial x}\right) d x
$$

In the following we will investigate $\dot{\mathrm{W}}_{\text {dis }}^{\mathrm{s}}$ and $\dot{\mathrm{W}}_{\mathrm{dis}}^{\mathrm{g} \cdot \mathrm{b}}$ separately. To evaluate $\left(\frac{\dot{w}_{d i s}}{U}\right)$, as remarked before, $k_{\text {tip }}$ and $2 \mathrm{~h}$ must be determined by solving the 
entire crack tip profile geometry. However, we may determine the order of magnitude by considering the limiting case of a unit cell in a rigid material containing a linear array of cracks with uniform center-to-center spacings $2 b$ and crack lengths $2 \mathrm{a}$ under a remote applied stress $\sigma_{a}$. Taking an extreme of rapid grain-boundary diffusion so that the normal stresses as well as the matter diffused from the crack surfaces are distributed uniformly along the grain boundary it is clear that $\sigma_{a} \cdot 2 b=\sigma_{t i p}(2 b-2 a)=2 \gamma_{s} \kappa_{t i p}(b-a), 2 b \dot{\delta}$ $=2 \mathrm{~h} \cdot 2 \mathrm{u}$, and $\sigma_{\mathrm{a}} \cdot 2 \mathrm{~b} \cdot \dot{\delta}=2 \dot{\mathrm{w}}_{\mathrm{dis}}^{\mathrm{s}}$ due to balances of force, mass and energy respectively. Combining these three equations and Eq. (30a) and solving for $K_{\text {tip }}$ yields

$$
K_{\text {tip }}=\frac{1+f}{h}\left(1-\gamma_{b} / 2 \gamma_{s}\right)
$$

where $f=(b-a) / a$ is a pure number. Substituting this expression for $K_{t i p}$ into $\mathrm{Eq} .(30 \mathrm{a})$ results in

$$
\frac{\dot{W}_{\text {dis }}^{s}}{u}=f\left(2 \gamma_{s}-\gamma_{b}\right)
$$

Although this equation is derived in a simple-minded fashion, it does reveal some important features in a general case. First, this expression shows that surface dissipative work is independent of the crack velocity $U$. Second, the absolute quantity is of the same order of magnitude as the Griffith energy. Indeed, by solving the linear diffusion equation (Fick's law) at the crack surfaces subject to the conditions of mass conservation, Chuang and Rice 12 gave an approximate closed form solution for the crack tip profile from which $K_{\text {tip }}$ is obtainable (see Eq. (24) of Ref. 12): 


$$
\begin{aligned}
\kappa_{\text {tip }} & =\left[2\left(1-\gamma_{b} / 2 \gamma_{s}\right)\right]^{1 / 2}\left(\mathrm{kT} / \mathrm{D}_{\mathrm{s}} \gamma_{\mathrm{s}} \Omega^{4 / 3}\right)^{1 / 3} u^{1 / 3} \\
& =\frac{2}{\mathrm{~h}}\left(1-\gamma_{\mathrm{b}} / 2 \gamma_{\mathrm{s}}\right)
\end{aligned}
$$

substituting Eq. (32) in Eq. (30a) leads to $f=1$ for this approximation. When evaluated precisely from a numerical technique, $f=1.1$ for the limiting case in which $\gamma_{b}=0$. In fact, $f$ lies between 1.0 and 1.1 depending upon $\theta_{t i p}$ for any material. Setting $f=1$ will suffice for all practical purposes. If we let $\theta_{\text {tip }}=20^{\circ}$ as is true for many crystalline materials, then setting $f=1$ will result in an error of approximately $5.7 \%$. (The error which would correspond to the worst case $\left(\theta_{\text {tip }}=0\right)$ is then $\left.9 \%\right)$.

We note in passing the interesting situation regarding the validity of Eq. (30a) in quasi-equilibrium cavities of Hull-Rimmer type not considered in the present paper. In that case, the version of $\mathrm{Eq}_{\mathrm{q}}$ (32) is $\mathrm{K}_{\mathrm{tip}}=$ $\left(1-\gamma_{b} / 2 \gamma_{s}\right) / h$ from a simple geometric sketch. Insertion of this expression for $\mathrm{K}_{\text {tip }}$ into Eq. (30a) leads to $\dot{\mathrm{w}}_{\mathrm{d} i \mathrm{~s}}^{\mathrm{s}}=0$. This result can be verified by Eq. (22) since quasi-equilibrium shapes imply uniform curvature everywhere resulting in grad $\mu_{s}=0$. Hence the applicability of Eq. (30a) for evaluation of $\dot{\mathrm{W}}_{\mathrm{dis}}^{\mathrm{s}}$ is established for a cavity of general shape.

Now, in addition to $\kappa_{t i p}$, the $\sigma_{y y}$ and $\delta$ field parameters at the grain boundary must be determined according to Eq. (30b) prior to computing the dissipative work incurred by grain-boundary diffusion. The governing equations were derived from the requirements that matter be conserved and Fick's law be satisfied in the grain boundary, and that the stress and strain fields so generated in the interior satisfy the-equilibrium and compatibility 
conditions respectively, and also satisfy boundary conditions at the crack planes and at the outer boundaries. The results are two differential equations with two unknown functions $\sigma_{y y}(x)$ and $\delta(x)$ as follows ${ }^{2}$ :

$$
\begin{aligned}
& \sigma_{y y}(x)=\frac{K}{\sqrt{2 \pi x}}+\frac{E}{4 \pi\left(1-v^{2}\right)} \int_{0}^{\infty} \frac{\partial \delta / \partial x^{\prime}}{x^{\prime}-x} \sqrt{\frac{x^{\prime}}{x}} d x^{\prime} \\
& \delta(x)=\frac{D_{b} \delta_{b} \Omega}{u k T} \frac{d \sigma_{y y}}{d x}
\end{aligned}
$$

subject to the initial conditions at the crack tip $(x=0)$ :

$$
\begin{aligned}
& \sigma_{\text {yy }}(0)=\left[2\left(1-\gamma_{b} / 2 \gamma_{s}\right)\right]^{1 / 2}\left(k T \gamma_{s}^{2} / D_{s} \Omega^{4 / 3}\right)^{1 / 3} u^{1 / 3} \\
& \sigma_{\text {yy }}^{\prime}(0)=2\left[2\left(1-\gamma_{b} / 2 \gamma_{s}\right)\right]^{1 / 2}\left[\left(D_{s} \gamma_{s}\right)^{1 / 3}(k T)^{2 / 3} / D_{b} \delta_{b} \Omega^{5 / 9}\right] u^{2 / 3}
\end{aligned}
$$

where $K$ is the applied stress intensity factor; $D_{s} \Omega^{1 / 3}$ and $D_{b} \delta_{b}$ are surface and grain boundary diffusivities respectively, and kT has its usual meaning. The first term on the RHS of Eq. (33a) is just the well-known K-field created by the applied stress and the second term represents the residual stresses generated by non-uniform matter deposition due to grain boundary diffusion. Eqs. (33b) and (33d) follow from Fick's law and Eq. (33c) from Eqs. (14a) and (32). Note that both initial conditions are dependent on the crack velocity. Typical solutions for $\sigma_{y y}(x)$ and $\delta(x)$ at a fixed crack growth rate are plotted schematically in Fig. 1. The reader is referred to Ref. 2 for detailed solutions. It was shown there that a grain boundary parameter I exists, where $L=\left[\pi E D_{b} \delta_{b} \Omega / 4\left(1-v^{2}\right) u k T\right]^{1 / 2}$ has a dimension of length and is a function of material properties, temperature and crack velocity, such that the size of the "diffusion zone" is confined to within 4L ahead of the moving crack tip 
wherein the influence of diffusion is significant. The resulting small diffusion zone implies that the applied $\mathrm{K}$ field is able to control the crack growth behavior. Mathematically, this is analogous to the small cohesive zone in the Dugdale-Baranblatt model or small-scale yielding zone in the linear elastic fracture mechanics.

Hence, there is a one-to-one correlation between $K$ and $u$ for $u>u_{\min }$ :

$$
\mathrm{K} / \mathrm{K}_{\mathrm{Gr}}=0.845\left[\left(\mathrm{u} / \mathrm{u}_{\min }\right)^{1 / 12}+\left(\mathrm{u} / \mathrm{u}_{\min }\right)^{-1 / 12}\right]
$$

where

$$
u_{\min }=8.13 \mathrm{D}_{\mathrm{s}}^{4} \Omega^{7 / 3}\left[\mathrm{E} /\left(1-u^{2}\right) \mathrm{D}_{\mathrm{b}} \delta_{\mathrm{b}}\right]^{3} / \mathrm{KT} \gamma_{\mathrm{s}}^{2}
$$

and $G=J \propto \mathrm{K}^{2}$

or $\quad G / G_{G r}=J / J_{G r}=\left(K / K_{G r}\right)^{2}$

$$
=0.714\left[\left(u / u_{\min }\right)^{1 / 12}+\left(u / u_{\min }\right)^{-1 / 12}\right]^{2}
$$

where the subscript $G r$ relates to an ideal Griffith crack.

Eq. (34a) is the resulting $K-U$ kinetic law applicable to steady state diffusional crack growth in a linear elastic solid obeying Ficks law. A plot of this equation shows that a threshold value of $\mathrm{K}$ equal to $1.69 \mathrm{~K}_{\mathrm{Gr}}$ exists below which the crack can not propagate. Moreover, since $U_{\text {min }}$ is a constant depending on material properties and temperature (see Eq. (34b)), and has a 
finite value, it is not possible to have a crack growing at a speed slower than $U_{\text {min }}$ unless another crack growth mechanism takes over. The consequence can be explained in terms of energy considerations given below. At this point, it might be mentioned that Speight et al. ${ }^{13}$ claimed that their results could be made to coincide with the kinetic equation given here (see Eq. (34a)). Unfortunately, their claim only corresponds to the second term of the RHS of Eq. (34a) which is negligible when $U \gg U_{\min }$, a range where slit-like growth mode is expected to be operative.

The main features of the energy release rate that occurs during diffusive growth of a sharp crack can be unveiled if we plot nondimensional G vs. $U$ from Eq. (35) as illustrated in Fig. 2 where the three components of $G$ are shown for an arbitrary velocity. For example, at the threshold point where $U=U_{\text {min }}$ an amount of energy $0.85 \mathrm{GGr}_{\mathrm{Gr}}$ goes to dissipative work in the grain boundary whereas $1.0 \mathrm{G}_{\mathrm{Gr}}$ each is spent on $\dot{\mathrm{F}}_{\mathrm{s}} / \mathrm{U}$ as well as on surface diffusion. For a higher crack velocity corresponding to a higher applied load, the first part increases following the curve while the last two parts remain fixed. However, when $U<U_{\text {min }}$, there is insufficient energy available to move the crack tip and, as a result, the crack will cease to grow. The current theory is unable to predict, under this condition, whether the crack tip will remain stationary or begin to heal.

Fuentes-Samaniego and $\mathrm{Nix}^{4}$ asserted that a steady state exists whenever the energy dissipation rate remains constant with respect to time, namely

$$
\frac{\mathrm{d}}{\mathrm{dt}}\left(\dot{\mathrm{W}}_{\mathrm{dis}}^{\mathrm{s}}+\dot{\mathrm{W}}_{\mathrm{dis}}^{\mathrm{g} \cdot \mathrm{b}} \cdot \mathbf{)}=0\right.
$$


Substituting the expression for $\dot{W}_{\mathrm{dis}}^{\mathrm{s}}$ and $\dot{\mathrm{w}}_{\mathrm{dis}}^{\mathrm{g} \cdot \mathrm{b}}$ from Eq. (5) into Eq. (36) and then applying Euler's equation derived from variational principles, they obtained several governing equations from the natural boundary conditions. Examination of these equations reveals that $k_{t i p}$ plays an important role in the crack growth behavior. This is a feature consistent with the current treatment (see Eqs. (25) and (26)). Evidently, an invariant dissipative work must be a necessary condition for the existence of a steady state. However, it needs to be proved that Eq. (36) is a sufficient condition as well. Otherwise, it is likely that a "steady state" defined purely by Eq. (36) may yield a crack velocity as well as a crack tip shape which are continuously changing with time.

\section{Conclusion}

The energy release rates that take place during steady state diffusional crack growth were derived in general from the principles of irreversible thermodynamics. For the sake of completeness, the strain energy contribution is included in the formulation. The resulting expression is valid regardless of the form of constitutive law or phenomenological equations that a material must follow, and can be decomposed into three individual components representing surface Griffith energy and dissipative work necessary to complete the processes of surface diffusion as well as grain-boundary diffusion. We then find that $G$ is related directly to the well known J-integral when it is formulated properly in the framework of finite deformation elasticity. 
Quantitative evaluations of each component were made for a special case based on a linearized theory where solutions of field parameters are readily available. The results showed that the strain energy contribution is negligible and that the surface diffusion component has values ranging between 1.0 and $1.1 \mathrm{G}_{\mathrm{Gr}}$, depending on material and is independent of the crack velocity. On the other hand, the grain boundary diffusion component is a function of the crack tip curvature as well as the J-integral, and is a monotonically increasing function with increasing crack velocities starting from a minimum value of 0.85 times the Griffith energy at $u=U_{\min }$. The total $\mathrm{G}$ is equal to $\mathrm{J}$ which then can be interpreted as (negative) mechanical work done by the grain boundary normal stresses on the thickening of the interface by mass transport. Finally, $G$ remains equal to $\left(1-v^{2}\right) K^{2} / E$ which means that, as in the case of linear elastic fracture mechanics, the applied stress intensity factor is the key parameter controlling diffusional crack growth in a linear elastic material.

\section{Acknowledgement}

The author is grateful to Professor James R. Rice of Harvard University for several stimulating discussions on nonequilibrium thermodynamic formalism.

\section{$\underline{\text { References }}$}

1. T.-J. Chuang, K. I. Kagawa, J. R. Rice and I. B. Sills, Acta Metallurgica 27 (1979) 265-284.

2. T.-J. Chuang, Journal of the American Ceramic Society 65 (1982) 93-103.

3. J. R. Rice and T.-J. Chuang, Journal of the American Ceramic Society 64 (1981) 46-53. 
4. R. Fuentes-Samaniego and W. D. Nix, Philosophical Magazine A 44 (1981) 601-612.

5. H. B. Callen, Thermodynamics, John Wiley \& Sons, Inc. New York (1960).

6. I. -W. Chen and A. S. Argon, Acta Metallurgica 29 (1981) 1759-1768.

7. R. M. Thomson, Chapter 23 in Physical Metallurgy, Ed. by R. W. Cahn and P. Haasen, North-Holland Publishing Co. Amsterdam 1983.

8. J. R. Rice and D. C. Drucker, International Journal of Fracture Mechanics 3 (1967) 19-27.

9. J. R. Rice, in Fracture Vol. II, Academic Press, Inc. New York, (1968) 191-311.

10. J. D. Eshelby, in Inelastic Behavior of Solids, McGraw-Hill, New York (1970) 77-115.

11. J. R. Rice, Journal of Applied Mechanics 35 (1968) 379-386.

12. T.-J. Chuang and J. R. Rice, Acta Metallurgica 21 (1973) 1625-1628.

13. M. V. Speight, W. B. Beere and G. Roberts, Materials Science and Engineering 36 (1978) 155-163. 



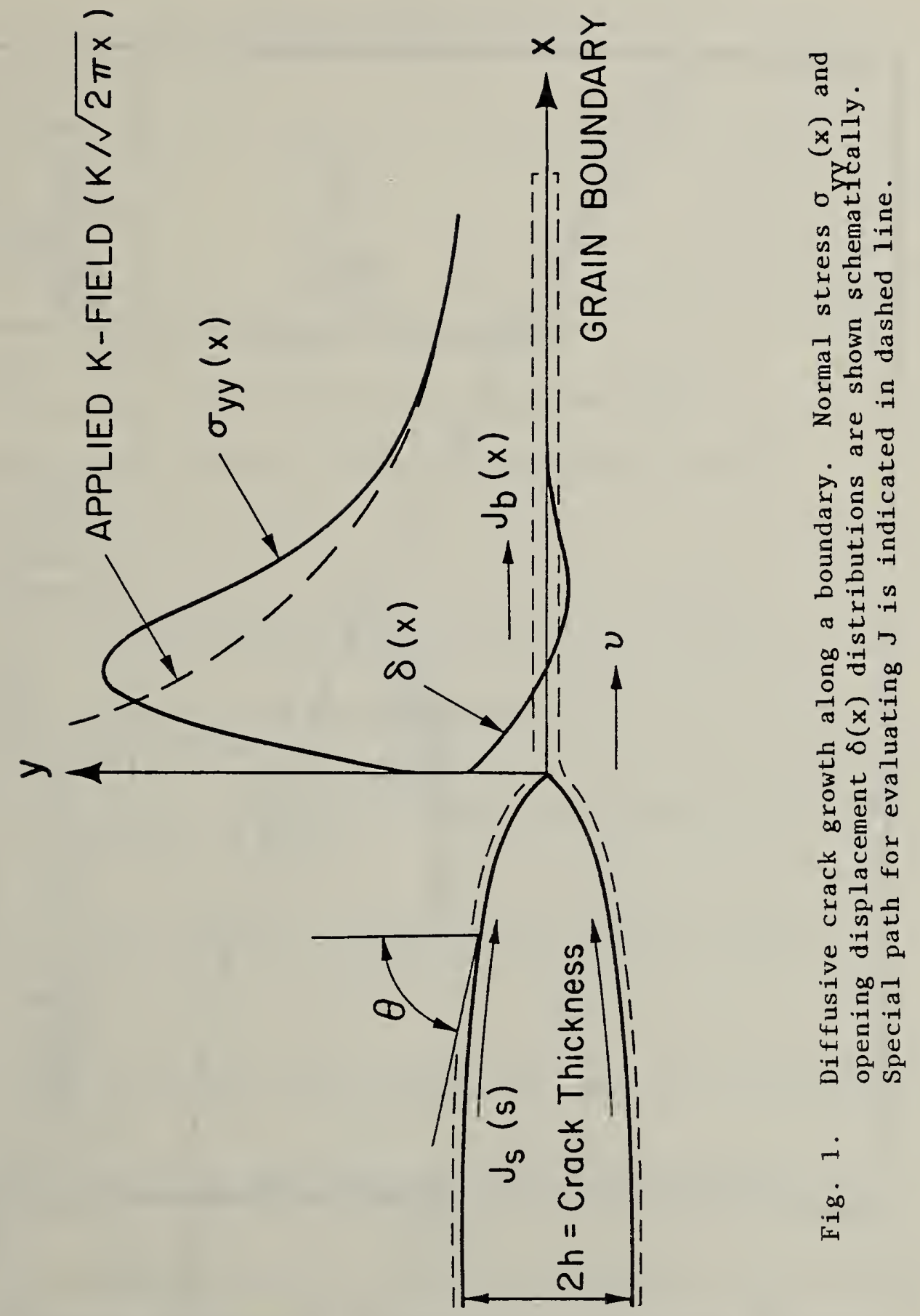




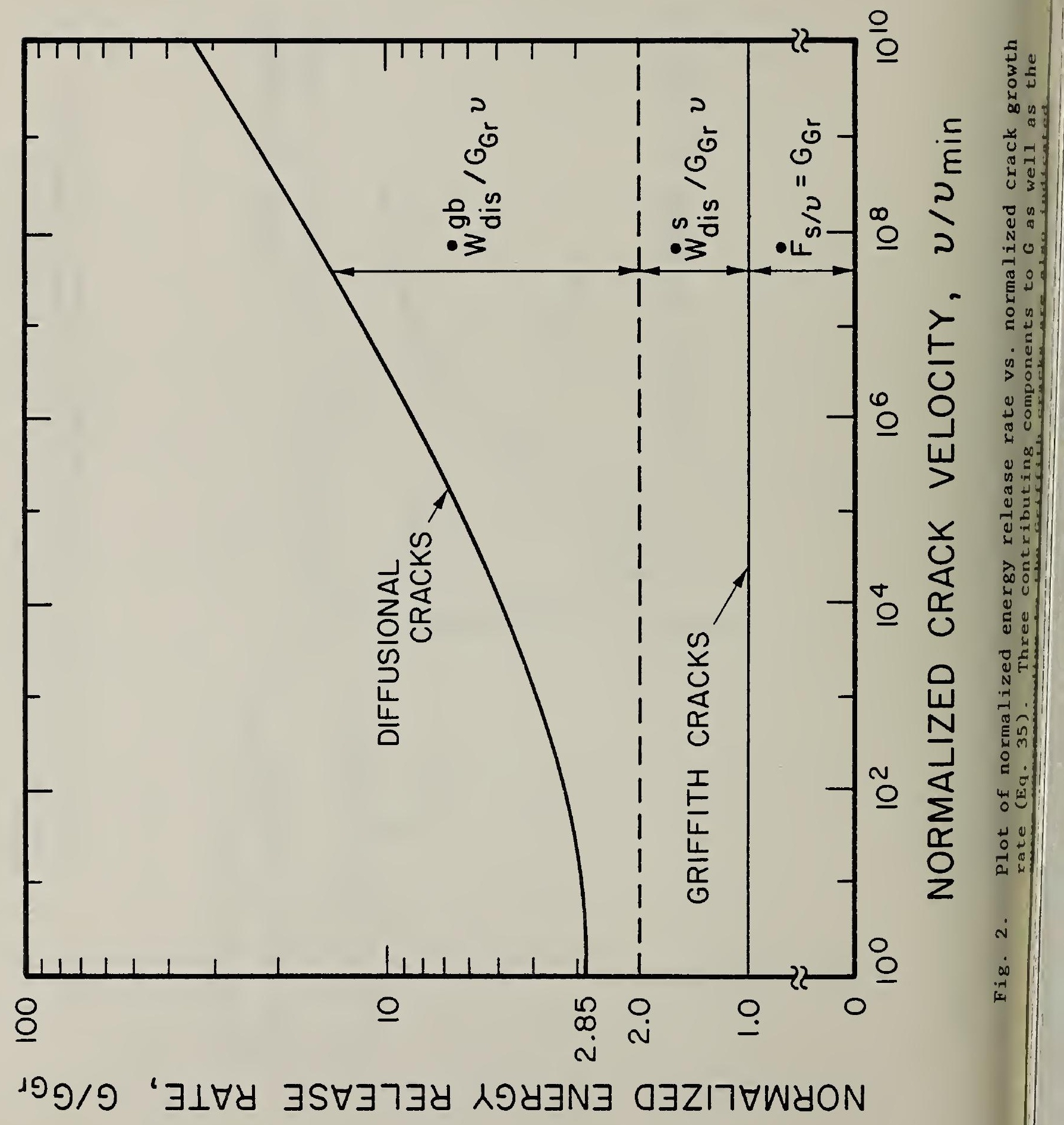


1. E. R. Fuller, Jr.

2. S. J. Schneider, Jr.

3. R. P. Reed

4. N. J. Tighe

5. J. B. Wachtman, Jr.

6. S. M. Wiederhorn

\section{EXTERNAL DISTRIBUTION}

7. Ames Laboratory, Iowa State University, Ames, Iowa 50011

0 . Buck

8-10. Argonne National Laboratory, 9700 S. Cass Avenue, Argonne, Illinois 60439

W. A. Ellingson

C. R. Kennedy

D. S. Kupperman

11. Battelle Columbus Laboratories, 505 King Avenue, Columbus, Ohio 43201

I. G. Wright

12. Bureau of Mines, Tuscaloosa Research Center, P.0. Box L, University, Alabama 35486

H. Heystek

13-14. Cornell University, Materials Sciences and Engineering Department, Bard Hall, Ithaca, NY 14853

C. -Y. Li

R. Raj

15-17. Electrical Power Research Institute, 3412 Hillview Avenue, P.0. Box 10412, Palo Alto, CA 94303

W. T. Bakker

R. I. Jaffee

J. Stringer

18. Exxon Research and Engineering Company, P.0. Box 101, Florham Park, New Jersey 07932

E. M. Anderson

19. Gas Research Institute, 8600 West Bryn Mawr Avenue, Chicago, Illinois 60631

V. L. Hill

20. IIT Research Institute, 10 West 35th Street, Chicago, Illinois 60616 S. A. Bortz

21. Lawrence Berkeley Laboratory, University of California, Berkeley, California 94720

A. G. Evans

22. Metal Properties Council, Inc., United Engineering Center, 345 E. 47 St., New York, NY 10017

A. 0 . Schaefer 
23. National Science Foundation, Division of Materials Research, Washington, DC 20550

B. A. Wilcox

24-27. Oak Ridge National Laboratory, P.O. Box X, Oak Ridge, Tennessee 37830

P. F. Becher

R. A. Bradley

P. T. Carlson

V. J. Tennery

28. Pennsylvania State University, College of Earth and Mineral Sciences, University Park, PA 16802

R. C. Bradt

29. Pennsylvania State University, Department of Materials Science and Engineering, University Park, PA 16802

30. Southwest Research Institute, 6220 Culebra Road, P.0. Drawer 29510, San Antonio, TX 78284

31 F. F. Lyle, Jr.

-32. Virginia Polytechnic Institute and State University, Blacksburg, VA 24601

J. J. Brown, Jr.

D. P. H. Hasselman

33-34. DOE, Office of Fossil Energy, 20 Massachusetts Avenue, NW Washington, DC 20545

S. J. Dapkunas

J. Fairbanks

35. DOE, Office of Basic Energy Sciences, 20 Massachusetts Avenue, NW Washington, DC 20545

L. Ianniello

36-37. DOE, Oak Ridge Operations Office, P.O. Box E, Oak Ridge, Tennessee 37830

E. E. Hoffman

Assistant Manager, Energy Research and Development

38. Technical Information Center, P.0. Box 62, Oak Ridge, Tennessee 37830 
4. TITLE AND SUBTITLE

\section{ON THE ENERGY-RELEASE RATE ASSOCIATED WITH DIFFUSIONAL CRACK GROWTH}

\section{AUTHOR(S)}

Tze-Jer Chuang

6. PERFORMING ORGANIZATION (If joint or other than NBS, see instructions)

NATIONAL BUREAU OF STANDARDS

DEPARTMENT OF COMMERCE

WASHINGTON, D.C. 20234

\section{SPONSORING ORGANIZATION NAME AND COMPLETE ADDRESS/STreet. Eity, StOTE, ZIP)}

7. Contract Grant No.

DE-A105-80CR20679

8. Type of Report \& Period Covered

AR\&TD Fossil Energy Materials Program

U.S. Department of Energy

10. SUPPLEMENTARY NOTES

Document describes a computer program; SF-185, FIPS Software Summary, is attached.

11. ABSTRACT (A 200-word or less factual summary of most significant information. If document includes a significant bibliography or literature survey. mention it here)

A general expression for the energy release rate $(G)$ that arise during steady state crack propagation by diffusion is derived from the standpoint of irreversible thermodynamics. Three contributing components of $G$ are identified: (i) the Griffith energy $\left(G_{G r}\right)$; ( $\left.i i\right)$ heat generated in the process of surface diffusion; and (iii) grain-boundary diffusion. Further, the total $G$ is shown to be directly related to the well-known J-integral if formulated in the framework of finite deformation elasticity. This expression for $G$ is valid in general even if the response of the material is not linear and the mass transport kinetics does not follow Fick's law. Quantitative evaluations of each component are made for the linear case where field solutions are available. The results show that component (ii) is approximately equal to $G_{G r}$ and is independent of the crack velocity (u) whereas component ( $i i j)$ is a monotonically increasing function with $G$ starting from $0.85 \mathrm{G}_{\mathrm{G}}$ when $\mathrm{U}$ is at threshold value; and that strain energy contributions can be neglected leading to $G=J=\left(1-v^{2}\right) K^{2} / E$. This means that $G$ is not primarily associated with the release of the strain energy rather, it stems mostly from the negative work done by the normal stresses on the thickening of the grain boundary due to non-uniform deposition of matter along it.

12. KEY WORDS (Six to twelve entries; alphabetical order: capitalize only proper names; and separate key words by semicolons) Creep cavitation; creep fracture; diffusional crack growth; energy release rate; high temperature fracture; J-integral; non-equilibrium thermodynamics; steady state crack propagation

13. AVAILABILITY

Xulimited

For Official Distribution. Do Not Release to NTIS

Order From Superintendent of Documents, U.S. Government Printing Office, Washington, D.C. 20402.
14. NO. OF PRINTED PAGES

\section{5}

15. Price 


